\title{
Hydrogen Adsorption on Palladium and Platinum Overlayers: DFT Study
}

\author{
Igor A. Pašti, ${ }^{1}$ Nemanja M. Gavrilov, ${ }^{1}$ and Slavko V. Mentus ${ }^{1,2}$ \\ ${ }^{1}$ Faculty of Physical Chemistry, University of Belgrade, Studentski trg 12-16, 11158 Belgrade, Serbia \\ ${ }^{2}$ Serbian Academy of Sciences and Arts, Knez Mihajlova 35, 11185 Belgrade, Serbia \\ Correspondence should be addressed to Igor A. Pašti, igor@ffh.bg.ac.rs
}

Received 15 February 2011; Accepted 13 May 2011

Academic Editor: Milan M. Jaksic

Copyright (C 2011 Igor A. Pašti et al. This is an open access article distributed under the Creative Commons Attribution License, which permits unrestricted use, distribution, and reproduction in any medium, provided the original work is properly cited.

\begin{abstract}
Hydrogen adsorption on twenty different palladium and platinum overlayer surfaces with (111) crystallographic orientation was studied by means of periodic DFT calculations on the GGA-PBE level. Palladium and platinum overlayers here denote either the Pd and Pt mono- and bilayers deposited over (111) crystallographic plane of Pd, Pt, Cu, and Au monocrystals or the (111) crystallographic plane of Pd and Pt monocrystals with inserted one-atom-thick surface underlayer of Pd, Pt, $\mathrm{Cu}$, and $\mathrm{Au}$. The attention was focused on the bond lengths, hydrogen adsorption energetics, mobility of adsorbed hydrogen, and surface reactivity toward hydrogen electrode reactions. Both the ligand and strain effects were considered, found to lead to a significant modification of the electronic structure of Pd and Pt overlayers, described through the position of the d-band center, and tuning of the hydrogen adsorption energy in the range that covers approximately $120 \mathrm{kJmol}^{-1}$. Mobility of hydrogen adsorbed on studied overlayers was found to be determined by hydrogen-metal binding energy. Obtained results regarding Pd layers on $\mathrm{Pt}(111)$ and $\mathrm{Au}(111)$ surfaces, in conjunction with some of the recent experimental data, were used to explain its electrocatalytic activity towards hydrogen evolution reaction.
\end{abstract}

\section{Introduction}

Hydrogen evolution/oxidation reaction (her/hor) is one of the most important electrochemical reactions from both theoretical and practical aspects. The search for new efficient electrocatalysts is motivated by the high price of platinum, which is known as the most efficient metal for her/hor [1]. This search necessary involves the investigation of her/hor on well-defined surfaces which was identified as essential in order to link the atomic-/molecular-level properties of the electrochemical interface to the macroscopic kinetic parameters.

The first insights in structural sensitivity of her/hor on $\mathrm{Pt}(\mathrm{hkl})$ were provided by Marković et al. $[2,3]$ and Barber et al. [4, 5]. The modification of known electrocatalyst surfaces was often undertaken to improve their catalytic activity. For instance, well-defined Pt surfaces, modified by palladium monolayers, have been used as model systems in electrocatalysis by Marković et al. [6]. They demonstrated that $\mathrm{Pd}_{\mathrm{ML}} / \mathrm{Pt}(111)$ surface was more active than the $\mathrm{Pt}(111)$ surface. Baldauf and Kolb [7] found that palladium grows pseudoisomorphically on $\mathrm{Au}(111)$ surface, while Kibler [8] recently demonstrated that catalytic performance of Pdmodified $\mathrm{Au}(111)$ electrodes depends on the number of deposited Pd layers. Kibler et al. [9] investigated the activity of several Pd-monolayer-modified surfaces demonstrating the linear relationship between the hydrogen desorption potential and the shift of the d-band center. The activity of platinum overlayers has been extensively studied primarily with respect to oxygen reduction reaction, and there is a lot of experimental data available in the literature $[10,11]$.

Greeley and Mavrikakis [12] emphasized the importance of the detailed knowledge of hydrogen chemistry of different surfaces, related closely to the practical importance of hydrogen. The authors screened promising solid catalysts by means of computational approach and reported the existence of the class of surfaces with low hydrogen binding energies and low barriers for hydrogen dissociation. Among the computational studies of hydrogen adsorption on well-defined surfaces, the work of Roudgar and Groß $[13,14]$ offers 
a deep insight into hydrogen chemisorption on Pd-modified $\mathrm{Au}(111)$ and $\mathrm{Au}(100)$ surfaces. The authors invoked both electronic structure of surfaces and geometrical effect in order to analyze adsorption and applied the obtained results to rationalize the reactivity of palladium nanoparticles supported by $\mathrm{Au}(111)$ surface, studied by Meier et al. [15]. Significant amount of theoretical results regarding electrocatalytic activity of well-defined surfaces was published recently by Schmickler and coworkers [16, 17]. For example, Santos et al. [17] reported the study of electrocatalytic activity of various Pd monolayers on different $\mathrm{M}(111)$ substrates and identified the main factors determining the electrocatalytic activity of the studied surfaces. They pointed out that catalytic performance of a particular nanostructure can be very different for oxidation versus reduction direction of any electrode reaction.

In the present work, we investigated systematically the hydrogen adsorption on 20 different palladium and platinum overlayer surfaces with a common (111) crystallographic orientation. Since the published experimental investigations indicate that, on platinum metals surfaces, her proceeds usually at high-coverage of strongly adsorbed hydrogen (underpotential deposited hydrogen, $H_{\text {upd }}$ ) $[1,18]$, we focused the attention on the high-coverage states, $\theta=1 \mathrm{ML}$. Finally, for selected surfaces, we discussed the correlation of calculated hydrogen adsorption energies to the experimentally determined catalytic activities.

\section{Computational Details}

The DFT calculations have been performed using PWscf code of the quantum Espresso distribution [19]. Ultrasoft pseudopotentials have been generated on the GGA-PBE level of approximation [20]. Basis set has been approximated by a set of plane waves with kinetic energy less than $435 \mathrm{eV}$, while charge density cutoff was 10 times higher. Equilibrium lattice parameters $\left(a_{0}\right)$ of clean metals considered in this study were obtained by fitting $E_{\mathrm{tot}}=f(a)$ curve, where $E_{\mathrm{tot}}$ is the total energy of bulk metal unit cell. Infinite surface has been obtained using periodic boundary conditions.

The (111) type surfaces have been modeled using $(1 \times 1)$ hexagonal supercell geometry with seven metal layers and $20 \AA$ thick vacuum layer separating periodic images along the $z$-direction of the cell. First 2D Brillouin zone has been sampled by $7 \times 7 \times 1$ set of $k$ points generated through the general Monckhorst-Pack scheme [21]. Hydrogen adsorption was allowed on the both sides of the slab in order to prevent electrostatic coupling of the periodic images. Both the slab and the adsorbate layer have been fully relaxed until the forces acting on atoms were below $0.05 \mathrm{eV}^{-1}$ The adsorption was analyzed at four distinct adsorption sites: fcc and hcp (threefold sites), bridge (twofold site) and atop (single-fold site). The energy of adsorption $\left(E_{\mathrm{ads}}\right)$ was calculated using the following equation:

$$
E_{\mathrm{ads}}=\frac{1}{2}\left(E_{\mathrm{slab}+2 \mathrm{H}}-\left(E_{\mathrm{slab}}+E_{\mathrm{H}_{2}}\right)\right) \text {, }
$$

where $E_{\text {slab }+2 \mathrm{H}}, E_{\text {slab }}$, and $E_{\mathrm{H}_{2}}$ are the total energy of the slab with one hydrogen atom adsorbed on each side of the slab, the total energy of the isolated slab, and the total energy of the isolated $\mathrm{H}_{2}$ molecule, respectively.

As previously discussed by Conway and Jerkiewicz [18], the presence of water has negligible effect on the H-Pt bond energy. Therefore, the water molecules were not included in the presented calculations. Strictly speaking, the described results correspond to the gas-phase adsorption of hydrogen, but we extended the results to hydrogen adsorption on metal-solution interface in order to discuss the catalytic performance of some of the surfaces included in this study (Section 3.3). The procedure described here is, however, common practice in computational studies of the problems related to electrochemistry [23].

\section{Results and Discussions}

3.1. Adsorption Trends: Equilibrium Geometries and Energetics. In this section, the dissociative hydrogen adsorption on transition and noble metal surfaces $(\mathrm{Cu}, \mathrm{Au}, \mathrm{Pt}$, or Pd), modified by palladium and platinum overlayers, was analyzed. The obtained equilibrium lattice parameters, used throughout the present work, were $3.63 \AA$ for $\mathrm{Cu}, 4.00 \AA$ for $\mathrm{Pd}, 4.01 \AA$ for $\mathrm{Pt}$, and $4.17 \AA$ for $\mathrm{Au}$. The Pd and $\mathrm{Pt}$ monolayers on other (111) metal surfaces are denoted here as $\mathrm{Pd}_{\mathrm{ML}} / \mathrm{M}(111)$ or $\mathrm{Pt}_{\mathrm{ML}} / \mathrm{M}(111)$. Depending on the lattice mismatch, these surfaces subject either compressive or tensile strain. The effects of strain on the electronic structure of the surfaces are already well documented in the literature [24]. However, electronic structure of surface atoms is inevitably modified by the presence of $\mathrm{M}$ atoms in the second layer. This is known as the ligand effect [25], which can be estimated separately from the strain effect by inserting a monolayer of other metal, $M_{\text {und }}$, under the first surface layer of the analyzed metal surface. This class of systems is denoted here as $\mathrm{M}_{\text {und }} / \mathrm{Pd}(111)$ or $\mathrm{M}_{\text {und }} / \mathrm{Pt}(111)$ (subscript "und" stands for underlayer; for such systems Greeley and Mavrikakis [12] used the term subsurface alloys). In order to observe the strain effects only, Mavrikakis et al. [26] proposed to introduce the lateral expansion or compression of the lattice. In the present study, the surfaces with double monolayers of palladium or platinum $\left(\left(\mathrm{Pd}_{\mathrm{ML}}\right)_{2} / \mathrm{M}(111)\right.$ or $\left.\left(\mathrm{Pt}_{\mathrm{ML}}\right)_{2} / \mathrm{M}(111)\right)$ were used in order to analyze the strain effects separated from the ligand effect, rather than applying the expansion of the metal lattice parallel to the surface. This is the way to make the obtained results comparable to the experimental data.

Table 1 summarizes the results relating to the electronic structure of studied surfaces (expressed through the position of the d-band center [27]), preferential adsorption sites, and hydrogen adsorption energy (the last one was calculated by means of (1).

The stability of the structure of the studied surfaces is a rather important issue, having in mind that surface electronic structure and their catalytic activity may vary with the variation of structural parameters. We address these problems to the studies available in the literature $[12,28]$. While some of the systems included in the present study are well known and could be easily realized experimentally, 
TABle 1: The d-band centers ( $\left.E_{\mathrm{d}-b a n d}\right)$, the preferential adsorption sites for $\mathrm{H}$ adsorption, and the energies of hydrogen adsorption $\left(E_{\text {ads }}\right)$ on studied surfaces. Both palladium and platinum surfaces are divided by bolded lines in three groups, depending on their exposition to the compressive strain (the first group), the absence of the surface strain (the second group), or the presence of the tensile strain (the third group).

\begin{tabular}{|c|c|c|c|c|c|c|c|}
\hline \multicolumn{4}{|c|}{ Palladium surface } & \multicolumn{4}{|c|}{ Platinum surfaces } \\
\hline Label & $E_{\text {d-band }} / \mathrm{eV}$ & Adsorption site & $E_{\text {ads }} / \mathrm{eV}$ & Label & $E_{\text {d-band }} / \mathrm{eV}$ & Adsorption site & $E_{\text {ads }} / \mathrm{eV}$ \\
\hline \multirow{2}{*}{$\mathrm{Pd}_{\mathrm{ML}} / \mathrm{Cu}(111)$} & \multirow{2}{*}{-2.42} & \multirow{2}{*}{ hcp } & \multirow{2}{*}{0.10} & $\mathrm{Pt}_{\mathrm{ML}} / \mathrm{Cu}(111)$ & -2.82 & atop & 0.40 \\
\hline & & & & $\left(\mathrm{Pt}_{\mathrm{ML}}\right)_{2} / \mathrm{Cu}(111)$ & -2.62 & atop & -0.05 \\
\hline \multirow{2}{*}{$\left(\mathrm{Pd}_{\mathrm{ML}}\right)_{2} / \mathrm{Cu}(111)$} & \multirow{2}{*}{-2.09} & \multirow{2}{*}{ fcc } & \multirow{2}{*}{-0.16} & $\mathrm{Pt}_{\mathrm{ML}} / \mathrm{Pd}(111)$ & -2.15 & $\mathrm{fcc}$ & -0.38 \\
\hline & & & & $\left(\mathrm{Pt}_{\mathrm{ML}}\right)_{2} / \mathrm{Pd}(111)$ & -2.42 & fcc & -0.39 \\
\hline $\operatorname{Pd}(111)$ & -1.71 & fcc & -0.48 & $\operatorname{Pt}(111)$ & -2.13 & fcc & -0.43 \\
\hline $\mathrm{Cu}_{\text {und }} / \mathrm{Pd}(111)$ & -1.76 & fcc & -0.40 & $\mathrm{Cu}_{\text {und }} / \operatorname{Pt}(111)$ & -2.14 & fcc & -0.18 \\
\hline $\mathrm{Pt}_{\text {und }} / \mathrm{Pd}(111)$ & -1.81 & fcc & -0.49 & $\mathrm{Pd}_{\text {und }} / \mathrm{Pt}(111)$ & -2.10 & $\mathrm{fcc}$ & -0.41 \\
\hline $\mathrm{Au}_{\mathrm{und}} / \mathrm{Pd}(111)$ & -1.63 & fcc & -0.46 & $\mathrm{Au}_{\mathrm{und}} / \mathrm{Pt}(111)$ & -1.91 & fcc & -0.39 \\
\hline $\mathrm{Pd}_{\mathrm{ML}} / \mathrm{Pt}(111)$ & -1.80 & fcc & -0.51 & Pt & -1.75 & $f_{c c}$ & -0.60 \\
\hline$\left(\mathrm{Pd}_{\mathrm{ML}}\right)_{2} / \mathrm{Pt}(111)$ & -1.68 & $\mathrm{fcc}$ & -0.51 & & 1.18 & & 0.00 \\
\hline $\mathrm{Pd}_{\mathrm{ML}} / \mathrm{Au}(111)$ & -1.37 & fcc & -0.60 & $\left(\mathrm{Pt}_{\mathrm{ML}}\right)_{2} / \mathrm{Au}(111)$ & -2.02 & fcc & -0.58 \\
\hline$\left(\mathrm{Pd}_{\mathrm{ML}}\right)_{2} / \mathrm{Au}(111)$ & -1.50 & fcc & -0.62 & & & & \\
\hline
\end{tabular}

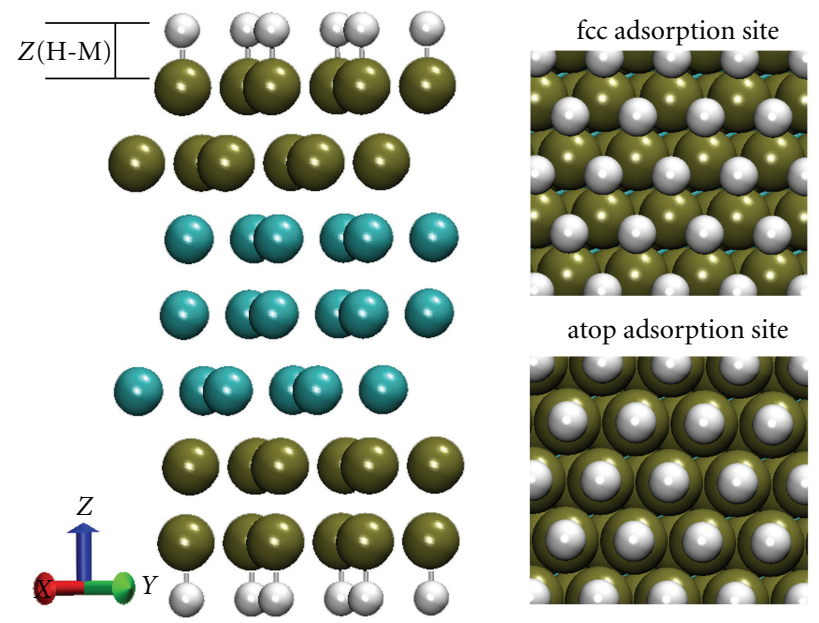

Figure 1: A side view of the metal slabs applied for hydrogen adsorption analysis and a perpendicular view of the surface covered with hydrogen adsorbed on fcc and atop site. For graphical representations, Visual Molecular Dynamics was used [22].

some of them, such as $\mathrm{M}_{\text {und }} / \mathrm{Pd}(111)$ and $\mathrm{M}_{\text {und }} / \mathrm{Pt}(111)$, are supposed only theoretically, for the purpose of analysis of the effects of electronic structure on the catalytic activity and on adsorption trends.

The vertical displacement between the centers of the adsorbed hydrogen atoms and the metal atoms in the first surface layer was used to characterize equilibrium geometry of adsorbed hydrogen on a given surface (adsorption height, as defined by Roudgar and Groß [13], Z(H-M), Figure 1). The parameter $Z(\mathrm{H}-\mathrm{M})$ was found to be the smallest for the adsorption on threefold sites, medium on the bridge sites, and largest for the adsorption on the atop sites. The other equilibrium structure parameter is the metal-hydrogen bond length $(d(\mathrm{H}-\mathrm{M}))$. The trend in bond lengths is opposite

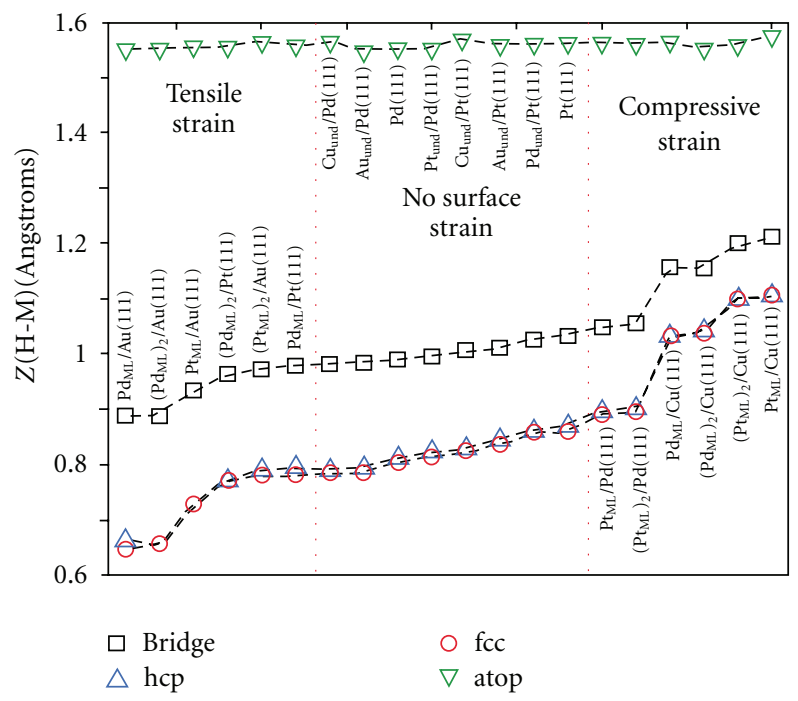

Figure 2: Calculated values of $Z(\mathrm{H}-\mathrm{M})$ on fcc (circles), hcp (up triangles), atop (down triangles), and bridge sites (squares).

to the trend in $Z(\mathrm{H}-\mathrm{M})$ distances. The bond length is the smallest for the atop adsorption (when the equation $Z(\mathrm{H}-\mathrm{M})$ $=d(\mathrm{H}-\mathrm{M})$ holds) while the largest one is for the adsorption on threefold sites. The interesting trends in calculated $Z(\mathrm{H}-$ $\mathrm{M})$ values were found observing the effects of surface strain on the equilibrium geometries (Figure 2). Namely, the $Z(\mathrm{H}$ $\mathrm{M})$ values of $\mathrm{H}$ atoms adsorbed on an atop site was found to be invariant to the type of the surface, with the relative variation not exceeding $0.5 \%$. Furthermore, the surfaces exposed to a tensile strain were found to have the smallest $Z(\mathrm{H}-\mathrm{M})$ distance. On the other hand, the surfaces exposed to a compressive strain have the highest $Z(\mathrm{H}-\mathrm{M})$. Between these two types of surfaces are the surfaces with no surface strain. The rationale for observed trends can be found in the work 


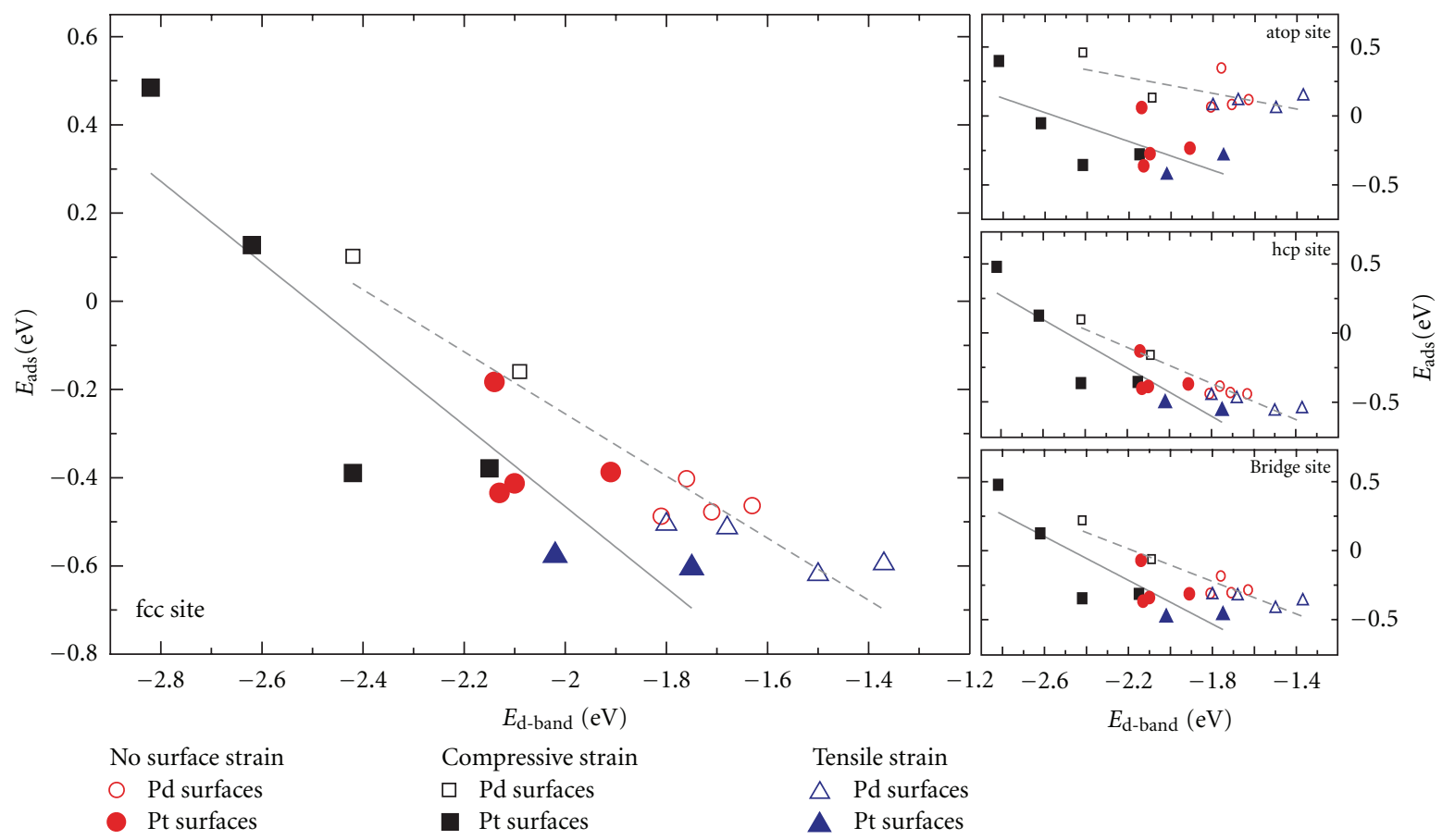

FIGURE 3: The energies of adsorption $\left(E_{\text {ads }}\right)$ plotted versus the position of the d-band center $\left(E_{\mathrm{d} \text {-band }}\right)$ for the studied surfaces. The surfaces with compressive strain are marked by squares; the surfaces with no strain are represented by circles, while the surfaces with tensile strain are marked by triangles. The empty symbols relate to Pd surfaces, while the full symbols relate to Pt surfaces. The best linear fits for platinum surfaces are given by solid lines, while the best linear fits for palladium surfaces are given by dashed lines. Positive values of $E_{\mathrm{ads}}$ mean the endothermic dissociative adsorption of $\mathrm{H}_{2}$ and vice versa.

of Roudgar and Groß [13]. These authors demonstrated, for Pd layers on Au surfaces, that the expansion of the crystal lattice parallel to the observed surface causes the decrease of $Z(\mathrm{H}-\mathrm{Pd})$, to preserve an ideal $\mathrm{H}-\mathrm{Pd}$ bond length related closely to the coordination of adsorbed $\mathrm{H}$ atom. Indeed, the site-dependent metal-hydrogen bond lengths calculated in the present study are almost identical for all studied surfaces. The average values of $d(\mathrm{H}-\mathrm{M})$ are $1.834 \AA$ for fcc site, $1.836 \AA$ for hcp site, $1.739 \AA$ for bridge site, and $1.558 \AA$ for atop site. The relative variation of $d(\mathrm{H}-\mathrm{M})$ over the entire group of studied surfaces (both Pd and Pt overlayer surfaces), for any of four adsorption sites, did not exceed $1 \%$. The differences in calculated $Z(\mathrm{H}-\mathrm{M})$ of the hydrogen atom adsorbed at different adsorption sites justify the fact that the ideal $\mathrm{H}$ $\mathrm{M}$ bond distance depends on the coordination of adsorbed $\mathrm{H}$ atom. Results presented here unambiguously confirm these reasoning for a wide range of surfaces. Location of the adsorbed hydrogen atom with respect to the surface might have some important consequences on the mobility of adatoms and catalytic performance of the particular surface, as will be discussed later.

Hydrogen adsorption energies are closely related to the catalytic activity of solid surfaces toward hor/her. Parsons demonstrated volcano type of dependence having the maximum around $\Delta G^{0}=0$, when logarithm of exchange current density for her was plotted versus standard Gibbs free energy of adsorption of $\mathrm{H}\left(\Delta G^{0}\right)$ [29]. This topic was reviewed in detail by Trasatti $[30,31]$. Hydrogen adsorption energies on surfaces studied in this paper cover a range of about $120 \mathrm{kJmol}^{-1}$, which is the result of substantial modification of electronic structure of studied surfaces $\left(E_{\mathrm{d}-\mathrm{band}}\right.$, Table 1$)$. Following the work of Hammer and Nørskov [27], the correlation between the adsorption energy and the position of the d-band center of the surface atoms is expected. Namely, the interaction of the adsorbate states with the metal d-states can be considered as a perturbation of the main adsorbate-substrate states, that is the interaction of adsorbate states with the substrate sp-states. The strength of the perturbation depends on (a) the fractional filling of substrate d-states $(f, f(\mathrm{Pt})<f(\mathrm{Pd})[24])$, (b) matrix elements for the interaction with the substrate $\left(V_{\mathrm{ad}}^{2}, V_{\mathrm{ad}}^{2}(\mathrm{Pt})>V_{\mathrm{ad}}^{2}(\mathrm{Pd})\right.$ [24]), and (c) the difference between the position of the adsorbate states and the substrate d-band center. As Pt and $\mathrm{Pd}$ atoms have different d-band fractional fillings and different matrix elements for interaction with adsorbates, $\mathrm{Pd}$ and Pt surfaces are not expected to fall onto the same $E_{\text {ads }}$ versus $E_{\text {d-band }}$ line. In the cases of multicoordinated sites, good correlations between $E_{\text {ads }}$ and $E_{\mathrm{d} \text {-band }}$ were observed, with $R^{2}$ close to 0.75 , while more pronounced scattering was evidenced in the case of atop adsorption site (Figure 3). As the theory of Hammer and Nørskov [27] does not treat all the factors able to contribute to the adsorbate-substrate bonding (e.g., the effects of the d-band widths are not considered), certain deviation from the linear relationship appears to be reasonable. It is interesting to note that adsorption parameters display higher sensitivity to the modification of 
surface electronic structure in the case of Pt overlayers than in the case of Pd overlayers. This may be explained by the fact that the slope of the dependence $E_{\text {ads }}$ versus $E_{\mathrm{d} \text {-band }}$ should be proportional to $(1-f) V_{\mathrm{ad}}^{2}$ [27], which is higher for platinum than for palladium.

Similarly to the equilibrium geometries, the surface activities depend strongly on the surface strain. The tensile strain shifts the d-band center towards the Fermi level, which is accompanied by negative shift in $E_{\text {ads }}$. The compressive strain lowers the position of d-band center and reduces the reactivity on the studied surfaces. Surfaces with no lateral strain display intermediate reactivity. Kibler [8] identified an upshift of $0.35 \mathrm{eV}$ of the d-band center of the first Pd layer in $\mathrm{Pd}_{\mathrm{ML}} / \mathrm{Au}(111)$ as the main reason of enhanced reactivity in comparison to the $\mathrm{Pd}(111)$ surface. Our calculation gave the up-shift of $0.34 \mathrm{eV}$. It is noteworthy (Table 1) that the introduction of the Au underlayer in $\operatorname{Pd}(111)$ surface causes destabilization (upshift) of palladium d-band center. Even more pronounced effect is observed in the case of $\mathrm{Pt}_{\mathrm{ML}} / \mathrm{Au}(111)$ and $\mathrm{Au}_{\text {und }} / \mathrm{Pt}(111)$ surfaces (Table 1).

Connected to the results presented here, Inderwildi et al. [32] analyzed modification of transition metal d-states in transition metal-coinage metal alloys surfaces in the case of Rh-Ag alloys and attributed an increased reactivity of the surfaces to the buildup of Rh d-states below the Fermi energy. Michaelides [33] pointed out possible importance of transition metal-coinage metal alloys in view of the fact that this class of materials can provide high surface reactivity and superior catalytic performance by subtle modification of surface composition.

3.2. Mobility of Adsorbed Hydrogen. As explained by Nilekar and coworkers [34], mobility of adsorbed species on transition metal surfaces, which is the function of $E_{\text {ads }}$, might play a key role in the transport of species during the catalytic reactions, growth of the surface nanostructures, and many other important processes. Roudgar and Groß [13] reported that the absolute values of $E_{\mathrm{ads}}$ are sensitive, but the adsorption trends on (111) surfaces are almost insensitive toward the change of surface coverage, allowing the analysis given below. To be more precise, although the absolute values of adsorption energies are expected to be affected by surface coverage, relative differences among different adsorption sites are expected to remain unaffected, as well as relative differences among different surfaces. Nevertheless, this is an interesting subject that should be subjected to detailed calculations. Following the work of Nilekar et al. [34], we found a linear relationship between the binding energy of initial/final state $\left(\mathrm{BE}_{\mathrm{IS}} / \mathrm{BE}_{\mathrm{FS}}\right)$ for surface diffusion and the binding energy of the transition state for surface diffusion $\left(\mathrm{BE}_{\mathrm{TS}}\right.$, Figure 4$)$. As the initial states preferential adsorption sites were taken to identify transition state, first plausible diffusion paths connecting high-symmetry sites on studied surfaces were established, and transition state was chosen to be the lowest adsorption site on the path connecting the initial and the final state. The final states are chosen to be adsorption sites located after the transition state along the diffusion path with a condition to be energetically more favorable than
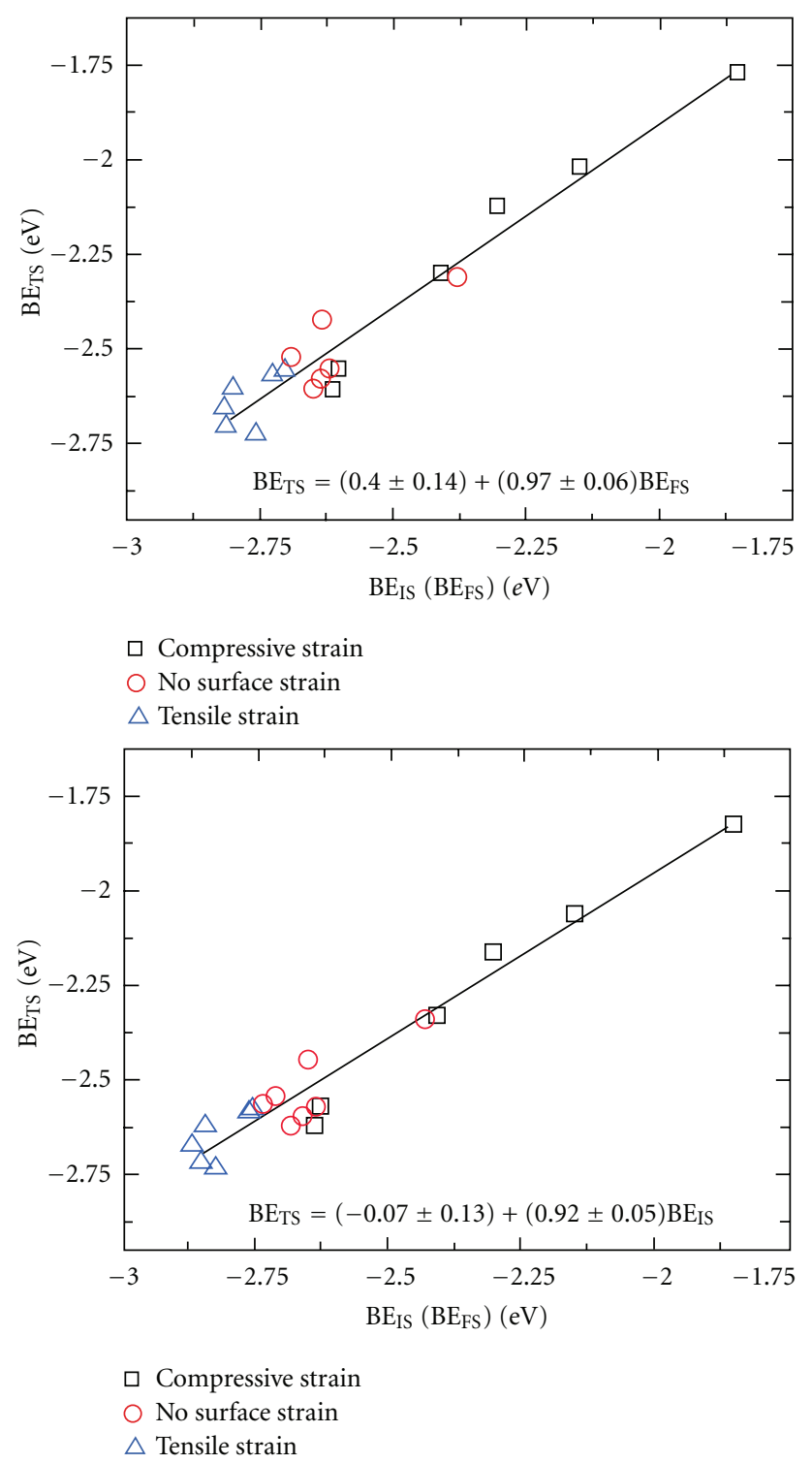

FIGURE 4: Lower pane: the binding energy of transition state for $\mathrm{H}$ diffusion $\left(\mathrm{BE}_{\mathrm{TS}}\right)$ plotted versus the binding energy of initial state $\left(\mathrm{BE}_{\mathrm{IS}}\right)$; upper pane: the binding energy of transition state for $\mathrm{H}$ diffusion ( $\mathrm{BE}_{\mathrm{TS}}$ ) plotted versus the binding energy of final state $\left(\mathrm{BE}_{\mathrm{FS}}\right)$. In order to calculate hydrogen binding energies we refer adsorbed state to the isolated $\mathrm{H}$ atom. Binding energy of $\mathrm{H}_{2}$ molecule was estimated here to amount to $4.50 \mathrm{eV}$.

transition state [35]. For most of the studied surfaces, the path runs from the fcc site (initial state), through the bridge site (transition state), to the neighboring hcp site (final state).

The results presented here for hydrogen mobility on palladium and platinum surfaces agree very well with results published by Nilekar et al. [34] for clean transition metal surfaces and seven adsorbates in either atomic or molecular state (25 substrate/adsorbate systems in total). This sounds with the finding of Nilekar et al. that potential energy barriers (calculated as $\mathrm{BE}_{\mathrm{TS}}-\mathrm{BE}_{\mathrm{IS}}$ ) for surface diffusion can be estimated as approximately one-tenth of binding energy of the initial state $\left(\mathrm{BE}_{\mathrm{IS}}\right)$ for surface diffusion (more precisely, 
$12 \%$ of BEIS ) [34]. Furthermore, detailed calculations of vibrational entropy of the initial and transition states for surface diffusion [34] showed that for the diffusion on transition metal surfaces there is no compensation between the activation energy and the preexponential factor. The authors showed that the preexponential factor for surface diffusion, proposed to amount to $10^{13} \mathrm{~s}^{-1}$, may be considered as a constant. Nevertheless, experimentally determined values may differ from this value by several orders of magnitude. To reconcile these opposing results, one may say that preexponential factors for surface diffusion are similar on different transition metals, without specifying its value, and that the potential energy barrier is what actually determines adsorbate mobility. These calculations, in general, indicate that the strength of adsorbate-substrate interaction determines the mobility of adsorbed species.

One may expect that the geometrical effects should display the impact on the mobility of adsorbed phase and contribute to certain scatter of diffusion energy barrier obtained by strict calculations based on the above-proposed scheme. Hence, the presented estimation of the potential energy barriers should be considered as a useful rule of thumb, as emphasized by Nilekar et al. [34]. Nevertheless, the range of hydrogen binding energies (Figure 4) indicates that the average diffusion barrier is approximately $25 \mathrm{kJmol}^{-1}$ (for the sake of comparison, the experimentally determined value for $\operatorname{Pt}(111)$ is $\left.30 \mathrm{kJmol}^{-1}[36]\right)$. This exceeds the thermal energy at room temperature by one order of magnitude and indicates reduced 2D mobility of adsorbed hydrogen. This is further supported by a large negative change of standard molar entropy accompanying the hydrogen adsorption on $\mathrm{Pt}$ surface [37].

Figure 4 demonstrates another important consequence of the surface strain. Namely, potential energy surface for hydrogen-surface interaction is expected to be rather rough, and the compressive strain makes it smooth. In connection with the $Z(\mathrm{H}-\mathrm{M})$ changes (Section 3.1, Figure 2), one may conclude that the applied tensile strain results in deep potential wells of stable adsorption sites and location of adsorbed hydrogen rather in the surface than on the surface. On contrary, compressive strain, smoothing the potential energy surface for hydrogen-metal interaction, squeezes out the adsorbed hydrogen (and exposes it to the solution phase, if one deals with the metal-solution interface).

3.3. Catalytic Performance of Selected Surfaces. Here we would like to make some correlation of presented results with the available experimental data relating to the catalytic behavior of precious metal surfaces toward the her/hor. As reviewed by Marković and Ross [1], $\mathrm{Pd}_{\mathrm{ML}} / \mathrm{Pt}(111)$ surface provides unique reactivity for her [6], being found at the apex of the volcano curve. Activation energy for her on this surface was found to be $9 \mathrm{kJmol}^{-1}$, which is half of the activation energy on clean $\mathrm{Pt}(111)$ surface, indicating that kinetics of her is significantly enhanced on $\mathrm{Pd}_{\mathrm{ML}} / \mathrm{Pt}(111)$. Compared to $\operatorname{Pt}(111)$ surface, on which her proceeds at $\theta=$ 0.67 ML of underpotentially adsorbed hydrogen $\left(\mathrm{H}_{\mathrm{upd}}\right)$ on $\mathrm{Pd}_{\mathrm{ML}} / \mathrm{Pt}(111)$, her proceeds at $\theta=1 \mathrm{ML}$ of $\mathrm{H}_{\text {upd }}[1]$.
Considering $\mathrm{H}_{\text {upd }}$ as an inhibitor species of the kinetics of her, Marković and Ross [1] emphasized the importance of hydrogen adsorption energetics for understanding of catalytic activity and suggested that this (paradoxical) activity of $\mathrm{Pd}_{\mathrm{ML}} / \mathrm{Pt}(111)$ surface can be explained by a strong $\left(\mathrm{Pd}_{\mathrm{ML}} / \mathrm{Pt}(111)\right)-\mathrm{H}_{\text {upd }}$ interaction and/or weaker $\mathrm{H}_{\text {upd }}-\mathrm{H}_{\text {upd }}$ interaction, compared to $\mathrm{Pt}(111)$ surface. The authors also stated that some amount of $\mathrm{H}_{\text {upd }}$ might sit in deeper potential wells of the threefold hollow sites, being more in the surface than on the surface. Our results suggest that the adsorption of $\mathrm{H}$ on $\mathrm{Pd}_{\mathrm{ML}} / \mathrm{Pt}(111)$ surface is favored by $8 \mathrm{kJmol}^{-1}$ compared to the clean $\mathrm{Pt}(111)$ surface, which is close to the change of the activation energy for her and supports the findings that the coverage of $\mathrm{H}_{\text {upd }}$ at Nernst potential is higher on $\mathrm{Pd}_{\mathrm{ML}} / \mathrm{Pt}(111)$ surface. The distance of adsorbed $\mathrm{H}$ atoms from the surface was found to be less in the case of $\mathrm{Pd}_{\mathrm{ML}} / \mathrm{Pt}(111)$ surface than in the case of $\mathrm{Pt}(111)$ surface (Figure 2). In addition, the reduced mobility of adsorbed $\mathrm{H}$ (Section 3.2) unambiguously confirms that adsorbed hydrogen sits in deeper potential wells (of fcc sites) on $\mathrm{Pd}_{\mathrm{ML}} / \mathrm{Pt}(111)$ surface than on $\mathrm{Pt}(111)$ surface. The strongly adsorbed $\mathrm{H}\left(\mathrm{H}_{\text {upd }}\right)$ on $\mathrm{Pd}_{\mathrm{ML}} / \mathrm{Pt}(111)$ leaves the atop sites available for the formation of weakly bound overpotentially deposited hydrogen $\left(\mathrm{H}_{\mathrm{opd}}\right)$, which is considered as an intermediate for her $[1,18]$. In contrast to $\mathrm{Pd}_{\mathrm{ML}} / \mathrm{Pt}(111)$ surface, clean $\mathrm{Pt}(111)$ surface has empty sites for $\mathrm{H}_{\mathrm{opd}}$ formation, and in this case, $\mathrm{H}_{\text {upd }}$ and $\mathrm{H}_{\text {opd }}$ are only formally distinguished by the potential range in which deposition occurs and not by the type of adsorption site [18]. Following the discussion given by Conway and Jerkiewicz [16], one may conclude that the high coverage of $\mathrm{H}_{\text {upd }}$ on $\mathrm{Pd}_{\mathrm{ML}} / \mathrm{Pt}(111)$ results in the shift of (kinetically relevant) $\Delta G^{0}$ of adsorbed $\mathrm{H}$ toward the theoretically significant $\Delta G^{0}=0$ condition proposed by Parsons [29]. This is, namely, the consequence of the fact that $\mathrm{H}_{\text {upd }}$ and $\mathrm{H}_{\text {opd }}$ occupy different adsorption sites.

The results presented here for Pd-modified $\mathrm{Au}(111)$ surface are in excellent agreement with the ones reported by Roudgar and Groß [13] for flat palladium nanoparticles on $\mathrm{Au}(111)$ surfaces [15]. Our results, together with the ones by Roudgar and Groß [13], suggest that hydrogen adsorption energy increases when going from both the $\operatorname{Pd}(111)$ and $\mathrm{Pd}_{\mathrm{ML}} / \mathrm{Au}(111)$ surfaces toward the $\left(\mathrm{Pd}_{\mathrm{ML}}\right)_{2} / \mathrm{Au}(111)$ surface. Recently, Kibler [8] reported the activity of different number of Pd layers deposited on $\mathrm{Au}(111)$ surface toward her. All, $\mathrm{Pd}(111), \mathrm{Pd}_{\mathrm{ML}} / \mathrm{Au}(111)$, and $\left(\mathrm{Pd}_{\mathrm{ML}}\right)_{2} / \mathrm{Au}(111)$ surfaces, display strong interaction with hydrogen and lie on one branch of the volcano curve [8, Figure 1]. This indicates that desorption of $\mathrm{H}_{2}$ is rate-determining step for her. Namely, the Heyrowsky reaction is known to be the rate-determining step on palladium [38] and can be reasonably assumed to hold for Pd-modified $\mathrm{Au}(111)$ surfaces. Qualitatively, the trends in exchange current densities of her on these three surfaces could be rationalized when the logarithm of exchange current density is plotted versus hydrogen chemisorption energy, referred to isolated $\mathrm{H}_{2}$ molecule (this value may be easily converted to $\mathrm{H}$-metal bond energy using $\mathrm{H}_{2}$ bond energy). We found here linear relationship between calculated $E_{\text {ads }}$ and experimentally determined $\log \left(j_{0}\right)$ values for her on the mentioned three surfaces (Figure 5). The decrease of the rate 


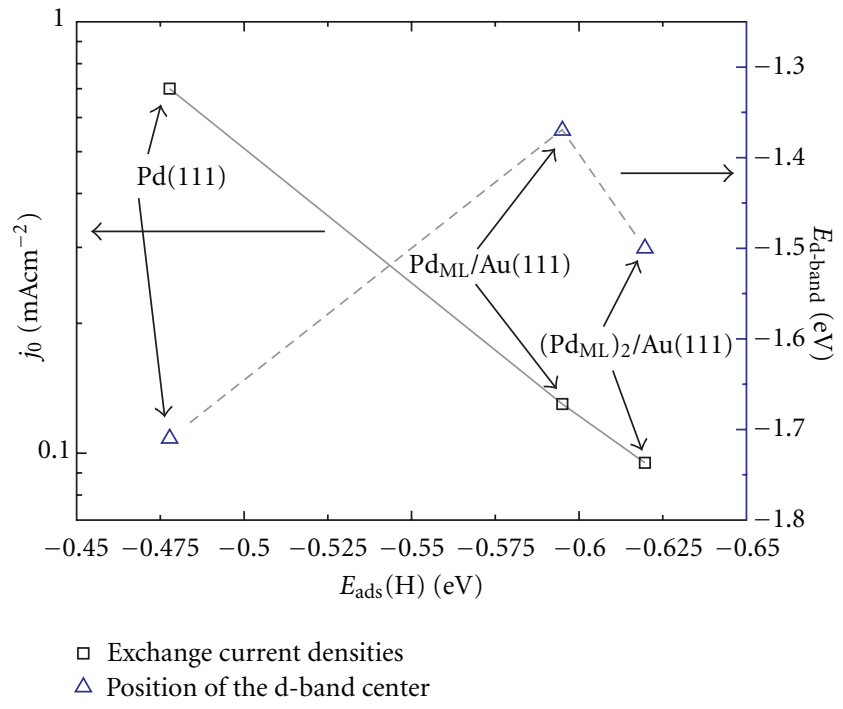

Figure 5: The literature data for standard exchange current densities $\left(j_{0}\right)$ for her on $\mathrm{Pd}(111)$ and Pd-modified $\mathrm{Au}(111)$ surfaces (squares) [8], and calculated positions of $E_{\mathrm{d} \text {-band }}$ (triangles) plotted versus calculated hydrogen adsorption energies.

of her may be correlated to strong $\mathrm{H}$-metal interaction, which limits the rate of $\mathrm{H}_{2}$ desorption. In Figure 5, also the position of $E_{\mathrm{d} \text {-band }}$ is presented as the function of $E_{\mathrm{ads}}$. Although $E_{\mathrm{d} \text {-band }}$ was in certain cases demonstrated as a valuable tool for establishing the electrocatalytic trends [39], this example demonstrates that one should be reserved in generalizations of this kind.

\section{Conclusions}

In the present study, the hydrogen adsorption on different palladium and platinum overlayer surfaces was studied by DFT method, from the aspects of the equilibrium geometries, the surface mobility, and the rate of electrode reactions. The effects of the surface strain and the electronic environment of surface layer (ligand effect) on the equilibrium geometries and adsorption energetics were estimated. It was shown that the mobility of adsorbed $\mathrm{H}$ may be directly correlated to the strength of adsorbate-substrate interaction. Presented results can be appended to the results of Nilekar and coworkers [34], showing that the relationship between the adsorption energies and the mobility of adsorbate holds not only for clean transition metal surfaces, but also for metal overlayers and subsurface alloys. In view of the previously evidenced absence of the compensation effect for surface diffusion on the transition metal surfaces [34], the mobility of adatoms may be considered to be completely determined by the strength of substrate-adsorbate interaction. In addition, the obtained results were used to analyze reactivity of $\mathrm{Pd}_{\mathrm{ML}} / \mathrm{Pt}(111)$ and $\mathrm{Pd}$-modified $\mathrm{Au}(111)$ surfaces toward the her/hor. For the explanation of the reactivity of $\mathrm{Pd}_{\mathrm{ML}} /$ $\mathrm{Pt}(111)$ surface, one has to take into account the adsorption energetics and the shape of the potential energy surface for the $\left(\mathrm{Pd}_{\mathrm{ML}} / \mathrm{Pt}(111)\right)-\mathrm{H}$ interaction. The increase in coverage of $\mathrm{H}_{\text {upd }}$ shifts the kinetically relevant $\Delta G^{0}$ for $\mathrm{H}$ adsorption toward the theoretically significant $\Delta G^{0}=0$ condition, proposed for her by Parsons [29]. This is considered as a consequence of the difference in the adsorption sites occupied by $\mathrm{H}_{\text {upd }}$ and $\mathrm{H}_{\text {opd }}$. However, for a rough explanation of the reactivity of $\mathrm{Pd}$-modified $\mathrm{Au}(111)$ surfaces, the adsorption energetics was found to be sufficient, through the finding that linear relationship exists between the $E_{\text {ads }}$ and the $\log \left(j_{0}\right)$. This linear dependence may be considered as a part of a branch of general volcano curve that correlates exchange current density for her to the hydrogen adsorption energy.

\section{Acknowledgments}

The authors are indebted to the Ministry of Science and Technological Development, Serbia, for the financial support under the Contract no. III45014. S.V. Mentus also acknowledges the support provided by the Serbian Academy of Science and Arts through the project "Electrocatalysis in the contemporary processes of energy conversion".

\section{References}

[1] N. M. Marković and P. N. Ross Jr., "Surface science studies of model fuel cell electrocatalysts," Surface Science Reports, vol. 45, no. 4-6, pp. 117-229, 2002.

[2] N. M. Marković, B. N. Grgur, and P. N. Ross Jr., "Temperaturedependent hydrogen electrochemistry on platinum low-index single-crystal surfaces in acid solutions," Journal of Physical Chemistry B, vol. 101, no. 27, pp. 5405-5413, 1997.

[3] N. M. Marković, S. T. Sarraf, H. A. Gasteiger, and P. N. Ross, "Hydrogen electrochemistry on platinum low-index singlecrystal surfaces in alkaline solution," Journal of the Chemical Society, vol. 92, no. 20, pp. 3719-3725, 1996.

[4] J. Barber, S. Morin, and B. E. Conway, "Specificity of the kinetics of $\mathrm{H}_{2}$ evolution to the structure of single-crystal $\mathrm{Pt}$ surfaces, and the relation between opd and upd H," Journal of Electroanalytical Chemistry, vol. 446, no. 1-2, pp. 125-138, 1998.

[5] J. H. Barber and B. E. Conway, "Structural specificity of the kinetics of the hydrogen evolution reaction on the low-index surfaces of Pt single-crystal electrodes in $0.5 \mathrm{M} \mathrm{dm}^{-3} \mathrm{NaOH}$," Journal of Electroanalytical Chemistry, vol. 461, no. 1-2, pp. 8089, 1999.

[6] N. M. Marković, C. A. Lucas, V. Climent, V. Stamenković, and P. N. Ross, "Surface electrochemistry on an epitaxial palladium film on $\mathrm{Pt}(111)$ : surface microstructure and hydrogen electrode kinetics," Surface Science, vol. 465, no. 1-2, pp. 103-114, 2000.

[7] M. Baldauf and D. M. Kolb, "A hydrogen adsorption and absorption study with ultrathin Pd overlayers on $\mathrm{Au}(111)$ and Au(100)," Electrochimica Acta, vol. 38, no. 15, pp. 2145-2153, 1993.

[8] L. A. Kibler, "Dependence of electrocatalytic activity on film thickness for the hydrogen evolution reaction of Pd overlayers on $\mathrm{Au}\left(\begin{array}{lll}1 & 1 & 1\end{array}\right)$," Electrochimica Acta, vol. 53, no. 23, pp. 68246828, 2008.

[9] L. A. Kibler, A. M. El-Aziz, R. Hoyer, and D. M. Kolb, “Tuning reaction rates by lateral strain in a palladium monolayer," Angewandte Chemie-International Edition, vol. 44, no. 14, pp. 2080-2084, 2005. 
[10] J. Zhang, M. B. Vukmirovic, K. Sasaki, A. U. Nilekar, M. Mavrikakis, and R. R. Adzic, "Mixed-metal Pt monolayer electrocatalysts for enhanced oxygen reduction kinetics," Journal of the American Chemical Society, vol. 127, no. 36, pp. 1248012481, 2005.

[11] V. Stamenković, T. J. Schmidt, P. N. Ross, and N. M. Marković, "Surface composition effects in electrocatalysis: Kinetics of oxygen reduction on well-defined $\mathrm{Pt}_{3} \mathrm{Ni}$ and $\mathrm{Pt}_{3} \mathrm{Co}$ alloy surfaces," Journal of Physical Chemistry B, vol. 106, no. 46, pp. 11970-11979, 2002.

[12] J. Greeley and M. Mavrikakis, "Alloy catalysts designed from first principles," Nature Materials, vol. 3, no. 11, pp. 810-815, 2004.

[13] A. Roudgar and A. Groß, "Local reactivity of thin Pd overlayers on Au single crystals," Journal of Electroanalytical Chemistry, vol. 548, pp. 121-130, 2003.

[14] A. Roudgar and A. Groß, "Local reactivity of metal overlayers: density functional theory calculations of Pd on Au," Physical Review B, vol. 67, no. 3, Article ID 033409, 4 pages, 2003.

[15] J. Meier, K. A. Friedrich, and U. Stimming, "Novel method for the investigation of single nanoparticle reactivity," Faraday Discussions, vol. 121, pp. 365-372, 2002.

[16] E. Santos, P. Quaino, P. F. Hindelang, and W. Schmickler, "Hydrogen evolution on a pseudomorphic Cu-layer on $\mathrm{Ni}(11$ 1) - a theoretical study," Journal of Electroanalytical Chemistry, vol. 649, no. 1-2, pp. 149-152.

[17] E. Santos, P. Quaino, and W. Schmickler, "On the electrocatalysis of nanostructures: monolayers of a foreign atom (Pd) on different substrates M(lll 11 1 ), Electrochimica Acta, vol. 55, no. 14, pp. 4346-4352, 2010.

[18] B. E. Conway and G. Jerkiewicz, "Relation of energies and coverages of underpotential and overpotential deposited $\mathrm{H}$ at Pt and other metals to the "volcano curve" for cathodic $\mathrm{H}_{2}$ evolution kinetics," Electrochimica Acta, vol. 45, no. 25-26, pp. 4075-4083, 2000.

[19] P. Giannozzi, S. Baroni, N. Bonini et al., "QUANTUM ESPRESSO: a modular and open-source software project for quantum simulations of materials," Journal of Physics Condensed Matter, vol. 21, no. 39, Article ID 395502, 2009.

[20] J. P. Perdew, K. Burke, and M. Ernzerhof, "Generalized gradient approximation made simple," Physical Review Letters, vol. 77, no. 18, pp. 3865-3868, 1996.

[21] H. J. Monkhorst and J. D. Pack, "Special points for Brillouinzone integrations," Physical Review B, vol. 13, no. 12, pp. 51885192, 1976.

[22] W. Humphrey, A. Dalke, and K. Schulten, "VMD: visual molecular dynamics," Journal of Molecular Graphics, vol. 14, no. 1, pp. 33-38, 1996.

[23] M. T. M. Koper, "Ab initio quantum-chemical calculations in electrochemistry," in Modern Aspects of Electrochemistry, Number 36, C. G. Vayenas, B. E. Conway, and R. E. White, Eds., chapter 2, Kluwer Academic/Plenum Publishers, New York, NY, USA, 2003.

[24] A. Ruban, B. Hammer, P. Stoltze, H. L. Skriver, and J. K. Nørskov, "Surface electronic structure and reactivity of transition and noble metals," Journal of Molecular Catalysis A, vol. 115, no. 3, pp. 421-429, 1997.

[25] J. R. Kitchin, J. K. Nørskov, M. A. Barteau, and J. G. Chen, "Role of strain and ligand effects in the modification of the electronic and chemical Properties of bimetallic surfaces," Physical Review Letters, vol. 93, no. 15, pp. 156801-4, 2004.

[26] M. Mavrikakis, B. Hammer, and J. K. Nørskov, "Effect of strain on the reactivity of metal surfaces," Physical Review Letters, vol. 81, no. 13, pp. 2819-2822, 1998.
[27] B. Hammer and J. K. Nørskov, "Electronic factors determining the reactivity of metal surfaces," Surface Science, vol. 343, no. 3, pp. 211-220, 1995.

[28] Y. Ma and P. B. Balbuena, "Pt surface segregation in bimetallic Pt3M alloys: a density functional theory study," Surface Science, vol. 602, no. 1, pp. 107-113, 2008.

[29] R. Parsons, "The rate of electrolytic hydrogen evolution and the heat of adsorption of hydrogen," Transactions of the Faraday Society, vol. 54, pp. 1053-1063, 1958.

[30] S. Trasatti, "The work function in electrochemistry," Advances in Electrochemical Science and Engineering, vol. 10, pp. 213321, 1977.

[31] S. Trasatti, in Handbook of Fuel Cells-Fundamentals, Technology and Applications, W. Vielstich, A. Lamm, and H. A. Gasteiger, Eds., vol. 2, p. 88, John Wiley \& Sons, Chichester, NY, USA, 2003.

[32] O. R. Inderwildi, S. J. Jenkins, and D. A. King, "When adding an unreactive metal enhances catalytic activity: $\mathrm{NO}_{x}$ decomposition over silver-rhodium bimetallic surfaces," Surface Science, vol. 601, no. 17, pp. L103-L108, 2007.

[33] A. Michaelides, "The unhappy marriage of transition and noble metal atoms: a new way to enhance catalytic activity? (A perspective on: "when adding an unreactive metal enhances catalytic activity: $\mathrm{NO}_{x}$ decomposition over silver-rhodium bimetallic surfaces" by O.R. Inderwildi, S.J. Jenkins, D.A. King)," Surface Science, vol. 601, no. 17, pp. 3529-3531, 2007.

[34] A. U. Nilekar, J. Greeley, and M. Mavrikakis, "A simple rule of thumb for diffusion on transition-metal surfaces," Angewandte Chemie-International Edition, vol. 45, no. 42, pp. 7046-7049, 2006.

[35] D. C. Ford, Y. Xu, and M. Mavrikakis, "Atomic and molecular adsorption on $\operatorname{Pt}\left(\begin{array}{lll}1 & 1 & 1\end{array}\right)$, , Surface Science, vol. 587, no. 3, pp. 159-174, 2005.

[36] E. G. Seebauer and L. D. Schmidt, "Surface diffusion of hydrogen on $\mathrm{Pt}(111)$ : laser-induced thermal desorption studies," Chemical Physics Letters, vol. 123, no. 1-2, pp. 129-133, 1986.

[37] B. E. Conway, H. Angerstein-Kozlowska, and W. B. A. Sharp, "Temperature and pressure effects on surface processes at noble metal electrodes. Part 1-entropy of chemisorption of $\mathrm{H}$ at Pt surfaces," Journal of the Chemical Society, Faraday Transactions 1, vol. 74, pp. 1373-1389, 1978.

[38] W. Schmickler and S. Trasatti, "Comment on "trends in the exchange current for hydrogen evolution" [J. Electrochem. Soc., 152, J23 (2005)]," Journal of the Electrochemical Society, vol. 153, no. 12, Article ID 053612JES, pp. L31-L32, 2006.

[39] V. R. Stamenkovic, B. S. Mun, M. Arenz et al., "Trends in electrocatalysis on extended and nanoscale Pt-bimetallic alloy surfaces," Nature Materials, vol. 6, no. 3, pp. 241-247, 2007. 


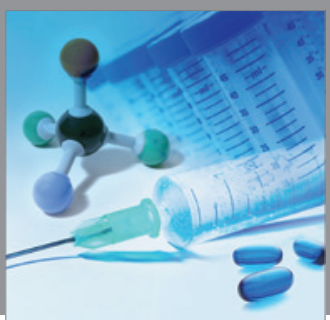

International Journal of

Medicinal Chemistry

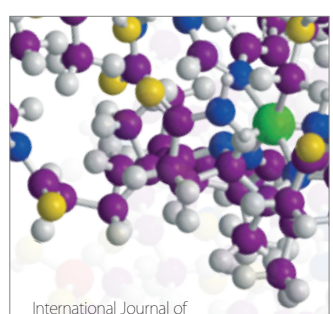

Carbohydrate Chemistry

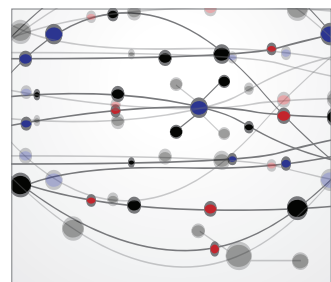

The Scientific World Journal
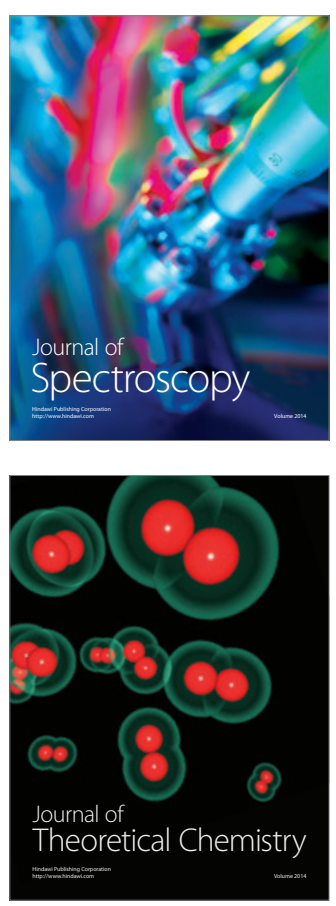
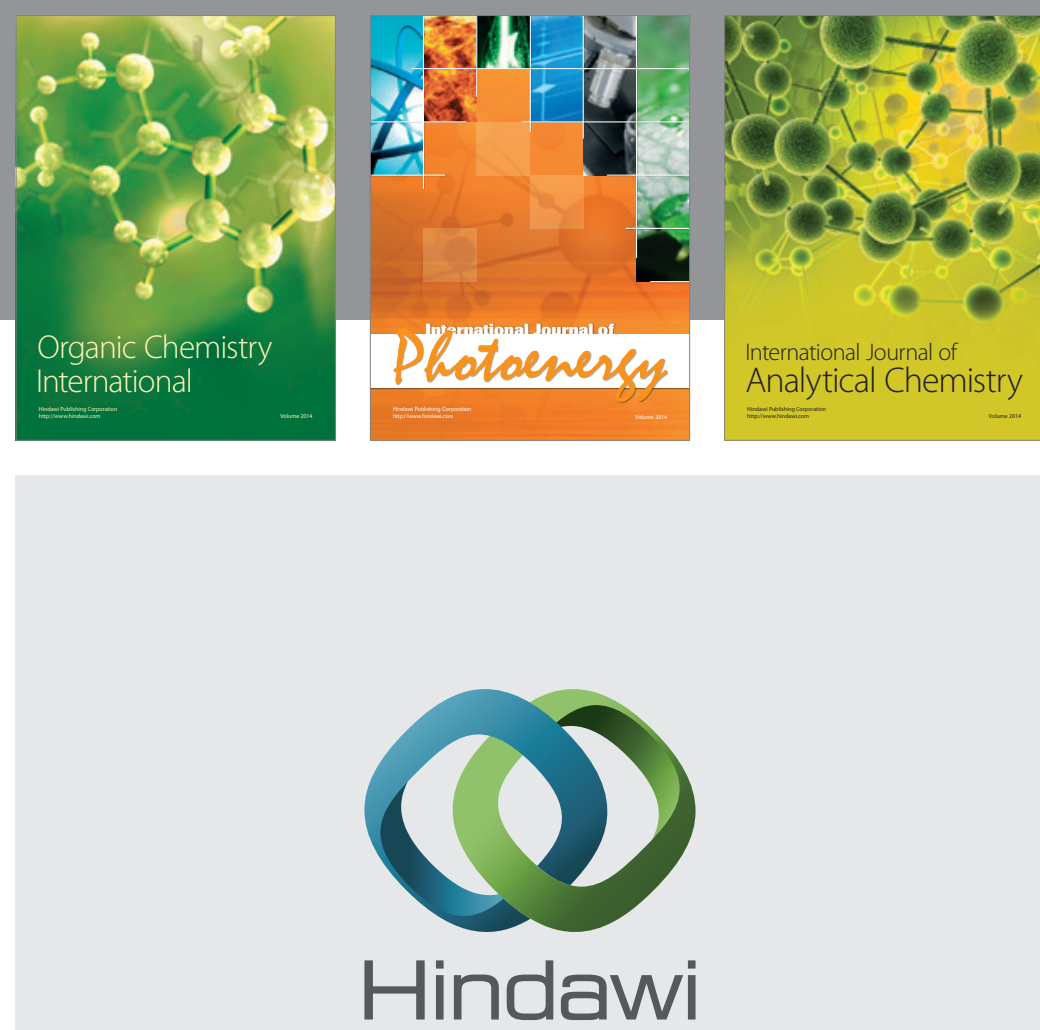

Submit your manuscripts at

http://www.hindawi.com
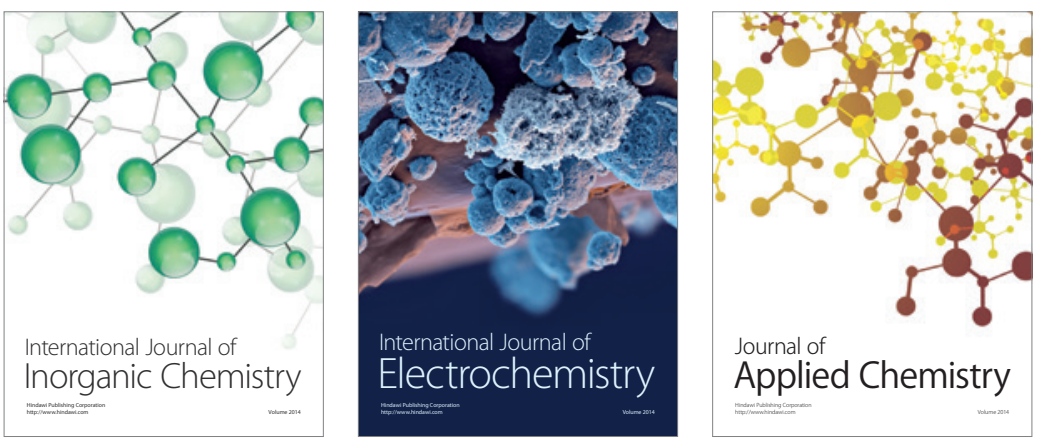

Journal of

Applied Chemistry
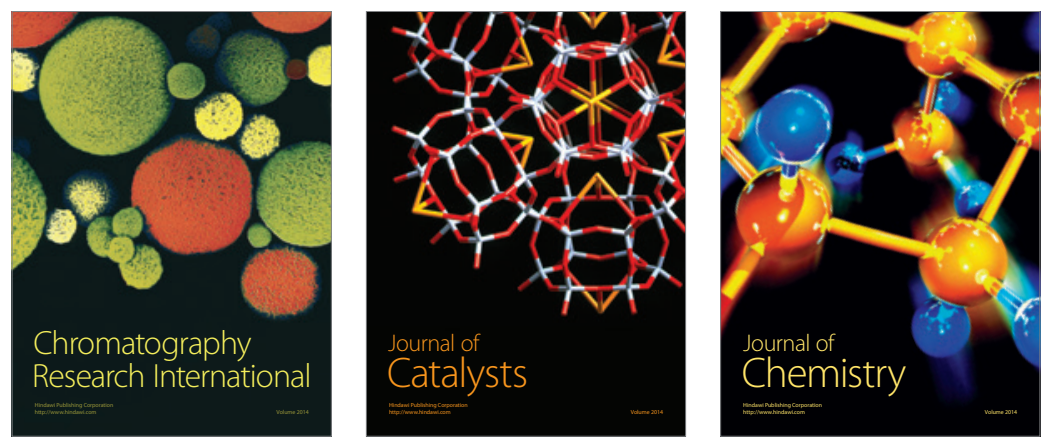
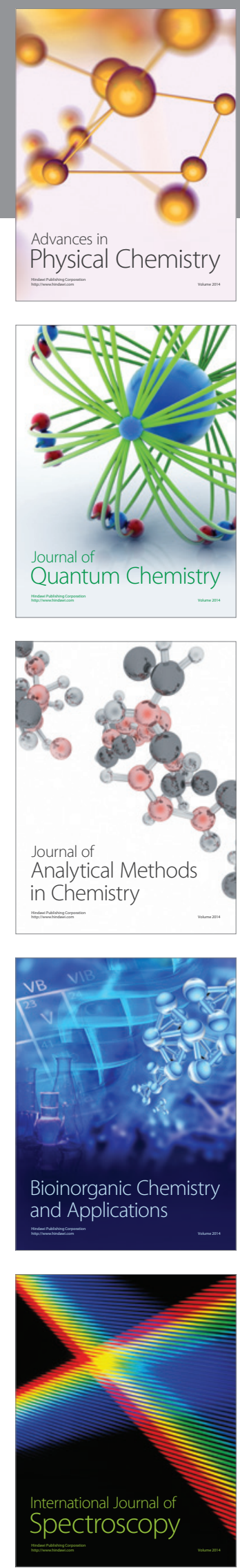